\title{
Convex Grid Drawings of Plane Graphs with Pentagonal Contours
}

\author{
Kazuyuki MIURA $^{\dagger \text { a) }}$,Member
}

SUMMARY In a convex drawing of a plane graph, all edges are drawn as straight-line segments without any edge-intersection and all facial cycles are drawn as convex polygons. In a convex grid drawing, all vertices are put on grid points. A plane graph $G$ has a convex drawing if and only if $G$ is internally triconnected, and an internally triconnected plane graph $G$ has a convex grid drawing on an $(n-1) \times(n-1)$ grid if either $G$ is triconnected or the triconnected component decomposition tree $T(G)$ of $G$ has two or three leaves, where $n$ is the number of vertices in $G$. An internally triconnected plane graph $G$ has a convex grid drawing on a $2 n \times 2 n$ grid if $T(G)$ has exactly four leaves. In this paper, we show that an internally triconnected plane graph $G$ has a convex grid drawing on a $6 n \times n^{2}$ grid if $T(G)$ has exactly five leaves. We also present an algorithm to find such a drawing in linear time. This is the first algorithm that finds a convex grid drawing of such a plane graph $G$ in a grid of polynomial size.

key words: algorithm, convex grid drawing, graph drawing, plane graph, triconnected

\section{Introduction}

Recently automatic aesthetic drawing of graphs has created intense interest due to their broad applications, and as a consequence, a number of drawing methods have come out [12]. The most typical drawing of a plane graph is a straight line drawing, in which all edges are drawn as straight line segments without any edge-intersection. A straight line drawing is called a convex drawing if every facial cycle is drawn as a convex polygon. One can find a convex drawing of a plane graph $G$ in linear time if $G$ has one [3], [4], [12].

A straight line drawing of a plane graph is called a grid drawing if all vertices are put on grid points of integer coordinates. This paper deals with a convex grid drawing of a plane graph. Throughout the paper we assume for simplicity that every vertex of a plane graph $G$ has degree three or more. Then $G$ has a convex drawing if and only if $G$ is "internally triconnected," that is, $G$ can be extended to a triconnected graph by adding a vertex in the outer face and joining it to all outer vertices [10], [13]. One may thus assume without loss of generality that $G$ is internally triconnected. If either $G$ is triconnected or the "triconnected component decomposition tree" $T(G)$ of $G$ has two or three leaves, then $G$ has a convex grid drawing on an $(n-1) \times(n-1)$ grid and such a drawing can be found in linear time, where $n$ is the number of vertices of $G$ [1], [2], [9]. Furthermore, an internally triconnected plane graph $G$ has a convex grid

Manuscript received March 26, 2013.

Manuscript revised July 24, 2013.

${ }^{\dagger}$ The author is with the Faculty of Symbiotic Systems Science, Fukushima University, Fukushima-shi, 960-1296 Japan.

a) E-mail: miura@sss.fukushima-u.ac.jp

DOI: 10.1587/transinf.E97.D.413

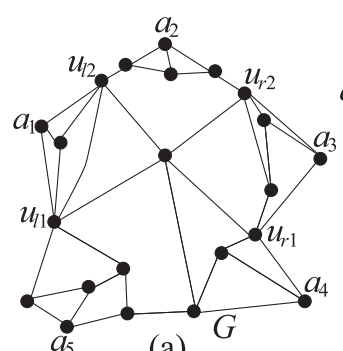

(a)

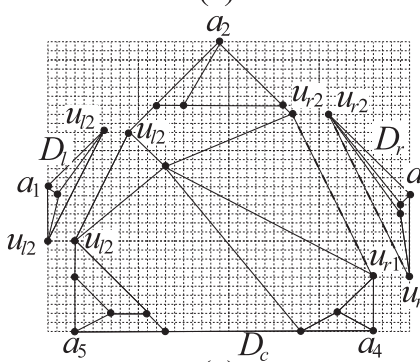

(c)

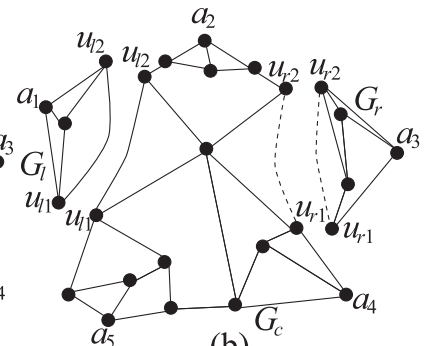

(b)

$a_{2}$

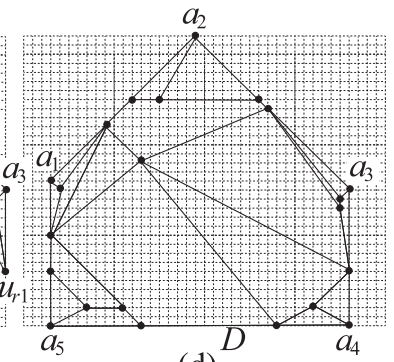

(d)
Fig. 1 (a) Plane graph $G$, (b) subgraphs $G_{c}, G_{l}$ and $G_{r}$, (c) convex grid drawings $D_{l}$ of $G_{l}, D_{r}$ of $G_{r}$ and $D_{c}$ of $G_{c}$, and (d) convex grid drawing $D$ of $G$.

drawing on a $2 n \times 2 n$ grid if $T(G)$ has exactly four leaves [8], [11], [15]. However, it has not been known whether $G$ has a convex grid drawing of polynomial size if $T(G)$ has five or more leaves. Figure 1 (a) depicts an internally triconnected plane graph $G$, Fig. 2 (c) the triconnected component decomposition tree $T(G)$ of $G$, which has five leaves $l_{1}, l_{2}, l_{3}, l_{4}$ and $l_{5}$.

In this paper, we show that an internally triconnected plane graph $G$ has a convex grid drawing on a $6 n \times n^{2}$ grid if $T(G)$ has exactly five leaves, and present an algorithm to find such a drawing in linear time. The algorithm is outlined as follows: we first divide a plane graph $G$ into a left subgraph $G_{l}$, a right subgraph $G_{r}$ and a center subgraph $G_{c}$ as illustrated in Fig. 1 (b) for the graph in Fig. 1 (a); we then construct convex grid drawings with triangular contours of $G_{l}$ and $G_{r}$ and construct a convex grid drawing with heptagonal contour of $G_{c}$ by a so-called shift method as illustrated in Fig. 1(c); we finally combine these three drawings to a convex grid drawing with pentagonal contour of $G$ as illustrated in Fig. 1 (d). This is the first algorithm that finds a convex grid drawing of such a plane graph $G$ in a grid of polynomial size.

The remainder of the paper is organized as follows. In Sect. 2 we give some definitions and known lemmas. In 
Sect. 3 we explain an algorithm for $G_{c}$. In Sect. 4 we explain an algorithm for $G_{l}$ and $G_{r}$. In Sect. 5 we present our convex grid drawing algorithm. Finally we conclude in Sect. 6.

\section{Preliminaries}

In this section, we give some definitions and known lemmas.

A $W \times H$ integer grid consists of $W+1$ regular vertical grid lines and $H+1$ regular horizontal grid lines, and has a rectangular contour. We call $W$ and $H$ the width and height of the integer grid, respectively. We denote by $W(D)$ the width of a minimum integer grid enclosing a grid drawing $D$ of a graph, and by $H(D)$ the height of $D$.

We denote by $G=(V, E)$ an undirected connected simple graph with vertex set $V$ and edge set $E$. We often denote the set of vertices of $G$ by $V(G)$ and the set of edges by $E(G)$. Throughout the paper we denote by $n$ the number of vertices in $G$. An edge joining vertices $u$ and $v$ is denoted by $(u, v)$. The degree of a vertex $v$ in $G$ is the number of neighbors of $v$ in $G$.

A plane graph $G$ divides the plane into connected regions, called faces. The infinite face is called an outer face, and the others are called inner faces. The boundary of a face is called a facial cycle. A cycle is represented by a clockwise sequence of the vertices in the cycle. We denote by $F_{\mathrm{o}}(G)$ the outer facial cycle of $G$. A vertex on $F_{\mathrm{o}}(G)$ is called an outer vertex, while a vertex not on $F_{\mathrm{o}}(G)$ is called an inner vertex. In a convex drawing of a plane graph $G$, all facial cycles must be drawn as convex polygons. The convex polygonal drawing of $F_{\mathrm{o}}(G)$ is called the outer polygon. We call a vertex of a polygon an apex in order to avoid the confusion with a vertex of a graph.

We call a vertex $v$ of a connected graph $G$ a cut vertex if its removal from $G$ results in a disconnected graph, that is, $G-v$ is not connected. A connected graph $G$ is biconnected if $G$ has no cut vertex. We call a pair $\{u, v\}$ of vertices in a biconnected graph $G$ a separation pair if its removal from $G$ results in a disconnected graph, that is, $G-\{u, v\}$ is not connected. A biconnected graph $G$ is triconnected if $G$ has no separation pair. A biconnected plane graph $G$ is internally triconnected if, for any separation pair $\{u, v\}$ of $G$, both $u$ and $v$ are outer vertices and each connected component of $G-\{u, v\}$ contains an outer vertex.

Let $G=(V, E)$ be a biconnected graph, and let $\{u, v\}$ be a separation pair of $G$. Then, $G$ has two subgraphs $G_{1}^{\prime}=\left(V_{1}, E_{1}^{\prime}\right)$ and $G_{2}^{\prime}=\left(V_{2}, E_{2}^{\prime}\right)$ satisfying the following two conditions (a) and (b).

(a) $V=V_{1} \cup V_{2}, V_{1} \cap V_{2}=\{u, v\}$; and

(b) $E=E_{1}^{\prime} \cup E_{2}^{\prime}, E_{1}^{\prime} \cap E_{2}^{\prime}=\emptyset,\left|E_{1}^{\prime}\right| \geq 2,\left|E_{2}^{\prime}\right| \geq 2$.

For a separation pair $\{u, v\}$ of $G, G_{1}=\left(V_{1}, E_{1}^{\prime}+(u, v)\right)$ and $G_{2}=\left(V_{2}, E_{2}^{\prime}+(u, v)\right)$ are called split graphs of $G$ with respect to $\{u, v\}$. The new edges $(u, v)$ added to $G_{1}$ and $G_{2}$ are called the virtual edges. Even if $G$ has no multiple edges, $G_{1}$ and $G_{2}$ may have. Dividing a graph $G$ into two split graphs $G_{1}$ and $G_{2}$ is called splitting. Reassembling the two split graphs $G_{1}$ and $G_{2}$ into $G$ is called merging. Merging

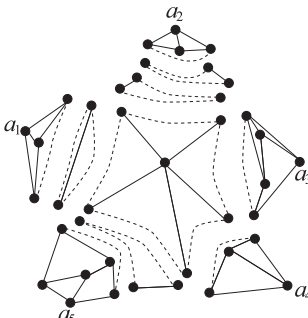

(a)

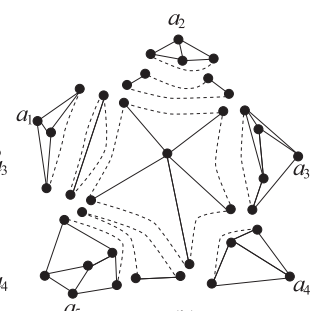

(b)

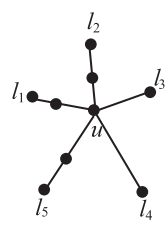

(c)
Fig. 2 (a) Split components of the graph $G$ in Fig. 1 (a), (b) triconnected components of $G$, and (c) a decomposition tree $T(G)$.

is the inverse of splitting. Suppose that a graph $G$ is split, the split graphs are split, and so on, until no more splits are possible, as illustrated in Fig. 2 (a) for the graph in Fig. 1 (a) where virtual edges are drawn by dotted lines. The graphs constructed in this way are called the split components of $G$. The graph in Fig. 1 (a) has ten split components as illustrated in Fig. 2 (a). The split components are of three types: a triconnected graph; a triple bond (i.e. a set of three multiple edges); and a triangle (i.e. a cycle of length three). The triconnected components of $G$ are obtained from the split components of $G$ by merging triple bonds into a bond and triangles into a ring, as far as possible, where a bond is a set of multiple edges and a ring is a cycle. Thus the triconnected components of $G$ are of three types:
(a) a triconnected graph;
(b) a bond; and
(c) a ring (which is not a triconnected graph, of course).

The split components of $G$ are not necessarily unique, but the triconnected components of $G$ are unique [7], [14]. Two triangles in Fig. 2 (a) are merged into a single ring, and hence the graph in Fig. 1 (a) has nine triconnected components as illustrated in Fig. 2 (b).

For a separation pair $\{u, v\}$ of $G$, two triconnected components $H_{i}$ and $H_{j}(i \neq j)$ are called the triconnected components of $G$ with respect to $\{u, v\}$ if it is possible to merge $H_{i}$ with $H_{j}$ at $\{u, v\}$. Let $T(G)$ be a tree such that each node corresponds to a triconnected component $H_{i}$ of $G$ and there is an edge $\left(H_{i}, H_{j}\right), i \neq j$, in $T(G)$ if and only if $H_{i}$ and $H_{j}$ are triconnected components with respect to the same separation pair, as illustrated in Fig. 2 (c). We call $T(G)$ a triconnected component decomposition tree or simply a decomposition tree of $G$ [7], [14]. We denote by $\ell(G)$ the number of leaves of $T(G)$. Then $\ell(G)=5$ for the graph $G$ in Fig. 1 (a). (See Fig. 2 (c).) If $G$ is triconnected, then $T(G)$ consists of a single isolated node and hence $\ell(G)=1$.

The following three lemmas are known.

Lemma 1: [7] A decomposition tree $T(G)$ of a graph $G$ can be found in linear time.

Lemma 2: [10] Let $G$ be a biconnected plane graph in which every vertex has degree three or more. Then the following three statements are equivalent to each other:

(a) $G$ has a convex drawing; 
(b) $G$ is internally triconnected; and

(c) both vertices of every separation pair are outer vertices, and a node of the decomposition tree $T(G)$ of $G$ has degree two if it is a bond.

Lemma 3: [10] If a plane graph $G$ has a convex drawing $D$, then the number of apices of the outer polygon of $D$ is no less than $\max \{3, \ell(G)\}$, and there is a convex drawing of $G$ whose outer polygon has exactly $\max \{3, \ell(G)\}$ apices.

Since $G$ is an internally triconnected simple graph and every vertex of $G$ has degree three or more, by Lemma 2 every leaf of $T(G)$ is neither a bond nor a ring but a triconnected graph. Lemmas 2 and 3 imply that if $T(G)$ has exactly five leaves, that is, $\ell(G)=5$ then the outer polygon of every convex drawing of $G$ must have five or more apices. Our algorithm finds a convex grid drawing of $G$ whose outer polygon is a pentagon and hence has exactly five apices, as illustrated in Fig. 1 (d).

In Sects. 3 and 4, we will present two algorithms to draw the center subgraph $G_{c}$, the left subgraph $G_{l}$ and the right subgraph $G_{r}$. (See Fig. 1 (c).) These algorithms use the following "canonical decomposition." Let $G=(V, E)$ be an internally triconnected plane graph, and let $V=$ $\left\{v_{1}, v_{2}, \cdots, v_{n}\right\}$. Let $v_{1}, v_{2}$ and $v_{n}$ be three arbitrary outer vertices appearing counterclockwise on $F_{\mathrm{o}}(G)$ in this order. We may assume that $v_{1}$ and $v_{2}$ are consecutive on $F_{\mathrm{o}}(G)$; otherwise, add a virtual edge $\left(v_{1}, v_{2}\right)$ to the original graph, and let $G$ be the resulting graph. Let $\Pi=$ $\left(U_{1}, U_{2}, \cdots, U_{m}\right)$ be an ordered partition of $V$ into nonempty subsets $U_{1}, U_{2}, \cdots, U_{m}$, where $U_{1} \cup U_{2} \cup \cdots \cup U_{m}=V$ and $U_{i} \cap U_{j}=\emptyset$ for any indices $i$ and $j, 1 \leq i<j \leq m$. We denote by $G_{k}, 1 \leq k \leq m$, the subgraph of $G$ induced by $U_{1} \cup U_{2} \cup \cdots \cup U_{k}$, and denote by $\overline{G_{k}}, 0 \leq k \leq m-1$, the subgraph of $G$ induced by $U_{k+1} \cup U_{k+2} \cup \cdots \cup U_{m}$. Clearly, $G_{k}=G-U_{k+1} \cup U_{k+2} \cup \cdots \cup U_{m}$ and $G=G_{m}=\overline{G_{0}}$. We say that $\Pi$ is a canonical decomposition of $G$ (with respect to vertices $v_{1}, v_{2}$ and $v_{n}$ ) if the following three conditions (cd1)-(cd3) hold:

(cd1) $U_{m}=\left\{v_{n}\right\}$, and $U_{1}$ consists of all the vertices on the inner facial cycle containing edge $\left(v_{1}, v_{2}\right)$.

(cd2) For each index $k, 1 \leq k \leq m, G_{k}$ is internally triconnected.

(cd3) For each index $k, 2 \leq k \leq m$, all the vertices in $U_{k}$ are outer vertices of $G_{k}$, and

(a) if $\left|U_{k}\right|=1$, then the vertex in $U_{k}$ has two or more neighbors in $G_{k-1}$ and has one or more neighbors in $\overline{G_{k}}$ when $k<m$, as illustrated in Fig. 3 (a); and

(b) if $\left|U_{k}\right| \geq 2$, then each vertex in $U_{k}$ has exactly two neighbors in $G_{k}$, and has one or more neighbors in $\overline{G_{k}}$, as illustrated in Fig. 3 (b).

Although the definition of a canonical decomposition above is slightly different from the one given in [2], they are effectively equivalent to each other. A canonical decomposition $\Pi=\left(U_{1}, U_{2}, \cdots, U_{11}\right)$ with respect to vertices $v_{1}, v_{2}$ and $v_{n}$ of the graph in Fig. 4 (a) is illustrated in Fig. 4 (b).

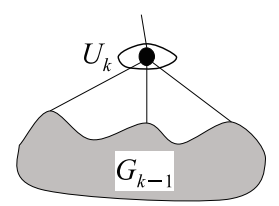

(a)

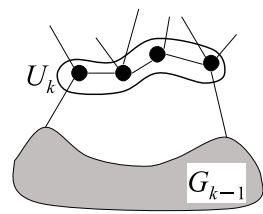

(b)
Fig. 3 (a) Graphs $G_{k}$ for which $\left|U_{k}\right|=1$ and (b) $\left|U_{k}\right| \geq 2$.

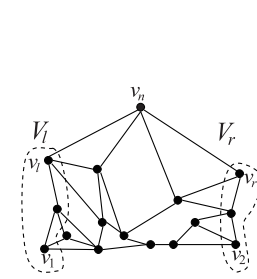

(a)

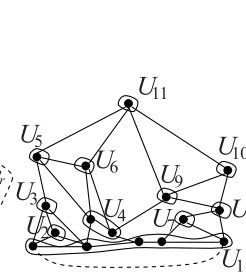

(b)

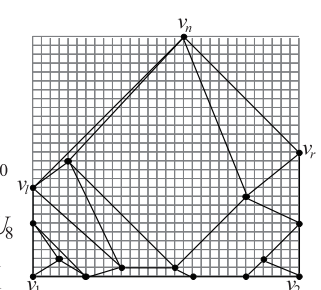

(c)
Fig. 4 (a) An internally triconnected plane graph $G$, (b) a canonical decomposition $\Pi$ of $G$, and (c) a pentagonal drawing $D$ of $G$.

By the condition (cd3), one may assume that all the vertices in $U_{k}, 1 \leq k \leq m$, consecutively appear clockwise on $F_{\mathrm{o}}\left(G_{k}\right)$. We number all vertices of $G$ by $1,2, \cdots, n$ so that they appear in $U_{1}, U_{2}, \cdots, U_{l}$ in this order. We call each vertex in $G$ by the number $i, 1 \leq i \leq n$. Thus one can define an order $<$ on the vertices in $G$. For a vertex $u, 1 \leq u \leq n-1$, we denote by $w^{*}(u)$ the largest neighbor of $u$.

The following lemma is known.

Lemma 4: [9] Assume that $G$ is an internally triconnected plane graph and $\ell(G) \leq 3$. Then one can find a canonical decomposition $\Pi$ of $G$ in linear time if $v_{1}, v_{2}$ and $v_{n}$ are chosen as follows.

Case $1: \ell(G)=3$.

In this case, from each of the three triconnected components corresponding to leaves of $T(G)$, we choose an arbitrary outer vertex of $G$ which is not a vertex of the separation pair of the component.

Case 2: $\ell(G)=2$.

In this case, we choose two vertices from the two leaves of $T(G)$, similarly to Case 1 above. We choose an arbitrary outer vertex of $G$ other than them as the third one.

Case 3: $\ell(G)=1$.

In this case, $G$ is triconnected. We choose three arbitrary outer vertices of $G$.

\section{Pentagonal Drawing}

Let $G$ be a plane graph having a canonical decomposition $\Pi=\left(U_{1}, U_{2}, \cdots, U_{m}\right)$ with respect to vertices $v_{1}, v_{2}$ and $v_{n}$, as illustrated in Fig. 4 (b). Miura et al. [11] give a linear-time algorithm, called the pentagonal drawing algorithm, to find a convex grid drawing of $G$ with a pentagonal outer polygon, as illustrated in Fig. 4 (c). The algorithm is based on the so-called shift methods given by Chrobak and Kant [2] and de Fraysseix et al. [5]. Unfortunately, this algorithm 
cannot find a convex gird drawing of an internally triconnected plane graph $G$ whose decomposition tree has four or more leaves. Thus we will modify the algorithm to find a convex grid drawing with heptagonal contour of the center subgraph $G_{c}$ of $G$ (whose decomposition tree has exactly three leaves), as illustrated in Fig. 1 (c), in Sect. 5.4.

We then outline the pentagonal drawing algorithm. Let $v_{l}$ be an arbitrary vertex on the path going from $v_{1}$ to $v_{n}$ clockwise on $F_{\mathrm{o}}(G)$, and let $v_{r}\left(\neq v_{l}\right)$ be an arbitrary vertex on the path going from $v_{2}$ to $v_{n}$ counterclockwise on $F_{\mathrm{o}}(G)$, as illustrated in Fig. 4 (a). Let $V_{l}$ be the set of all vertices on the path going from $v_{1}$ to $v_{l}$ clockwise on $F_{\mathrm{o}}(G)$, and let $V_{r}$ be the set of all vertices on the path going from $v_{2}$ to $v_{r}$ counterclockwise on $F_{\mathrm{o}}(G)$. Our algorithm finds a convex grid drawing of $G$ whose outer polygon is a pentagon with apices $v_{1}, v_{2}, v_{r}, v_{n}$ and $v_{l}$, as illustrated in Fig. 4 (c).

More precisely, the pentagonal drawing algorithm finds an "inner convex" grid drawing $D_{k}$ for each $k, 1 \leq k \leq m$, in which all inner facial cycles are convex polygons. Let $F_{\mathrm{o}}\left(G_{k-1}\right)=w_{1}, w_{2}, \cdots, w_{t}, w_{1}=v_{1}, w_{t}=v_{2}$. Let $w_{f}$ be the vertex with the maximum index $f$ among all the vertices $w_{i}$, $1 \leq i \leq t$, on $F_{\mathrm{o}}\left(G_{k-1}\right)$ that are contained in $V_{l}$. Let $w_{g}$ be the vertex with the minimum index $g$ among all the vertices $w_{i}$, $1 \leq i \leq t$, on $F_{\mathrm{o}}\left(G_{k-1}\right)$ that are contained in $V_{r}$. Of course, $1 \leq f<g \leq t$. We denote by $\angle w_{i}$ the interior angle of apex $w_{i}$ of the outer polygon of $D_{k-1}$. We call $w_{i}$ a convex apex of the polygon if $\angle w_{i}<180^{\circ}$. We denote the current position of a vertex $v$ by $P(v) ; P(v)$ is expressed by its $x$ - and $y$-coordinates of $(x(v), y(v))$. The drawing $D_{k}$ of $G_{k}$ satisfies the following six conditions (sh1)-(sh6).

(sh1) $P\left(w_{1}\right)=(0,0)$ and $P\left(w_{t}\right)=\left(2\left|V\left(G_{k-1}\right)\right|-2,0\right)$.

$(\operatorname{sh} 2) x\left(w_{1}\right)=x\left(w_{2}\right)=\cdots=x\left(w_{f}\right), x\left(w_{f}\right)<x\left(w_{f+1}\right)<$ $\cdots<x\left(w_{g}\right), x\left(w_{g}\right)=x\left(w_{g+1}\right)=\cdots=x\left(w_{t}\right)$, where $x\left(w_{i}\right)$ is the $x$-coordinate of $w_{i}$.

(sh3) Every edge $\left(w_{i}, w_{i+1}\right), f \leq i \leq g-1$, has slope $-1,0$, or 1.

(sh4) The Manhattan distance between any two grid points $w_{i}$ and $w_{j}, f \leq i<j \leq g$, is an even number.

(sh5) Every inner face of $G_{k-1}$ is drawn as a convex polygon.

(sh6) Vertex $w_{i}, f+1 \leq i \leq g-1$, has one or more neighbors in $\overline{G_{k-1}}$ if $w_{i}$ is a convex apex.

The following lemma is known.

Lemma 5: [11] For a plane graph $G$ having a canonical decomposition $\Pi=\left(U_{1}, U_{2}, \cdots, U_{m}\right)$ with respect to $v_{1}, v_{2}$ and $v_{n}$, the pentagonal drawing algorithm yields a convex grid drawing of $G$ on a $W \times H$ grid with $W=2 n-2$ and $H \leq n^{2}-n-2$ in linear time.

\section{Triangular Drawing}

Let $G$ be a plane graph having a canonical decomposition $\Pi=\left(U_{1}, U_{2}, \cdots, U_{m}\right)$ with respect to vertices $v_{1}, v_{2}$ and $v_{n}$, as illustrated in Fig. 5(b). In this section, we present

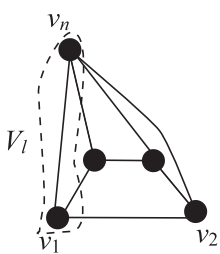

(a)

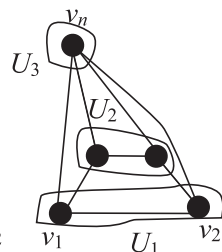

(b)

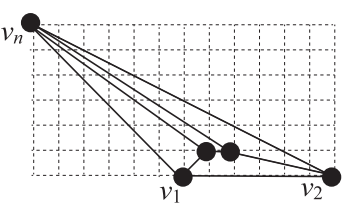

(c)
Fig. 5 (a) An internally triconnected plane graph $G$, (b) a canonical decomposition $\Pi$ of $G$, and (c) a triangular drawing $D$ of $G$.

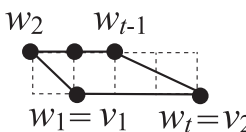

(a)

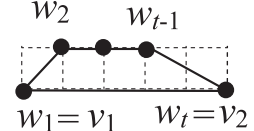

(b)

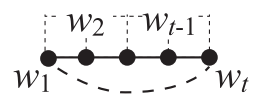

(c)
Fig. 6 Drawings $D_{1}$ of $G_{1}$ (a), (b) for Case 1 and (c) for Case 2.

a linear-time algorithm, called a triangular drawing algorithm, to find a convex grid drawing of $G$ with a triangular outer polygon. This algorithm is based on the algorithm in [2], and will be used by our convex grid drawing algorithm to draw the left subgraph $G_{l}$ and the right subgraph $G_{r}$ of $G$ in Sects. 5.2 and 5.3, respectively.

Our algorithm finds a convex grid drawing of $G$ whose outer polygon is a triangle with apices $v_{1}, v_{n}$ and $v_{2}$; the side $v_{1} v_{2}$ is horizontal, the side $v_{1} v_{n}$ has slope -1 , and the side $v_{2} v_{n}$ has slope $-1 / 2$, respectively, as illustrated in Fig. 5 (c). Let $V_{l}$ be the set of all vertices on the path going from $v_{1}$ to $v_{n}$ clockwise on $F_{\mathrm{o}}(G)$, as illustrated in Fig. 5 (a).

We first obtain a drawing $D_{1}$ of the subgraph $G_{1}$ of $G$ induced by all vertices in $U_{1}$. Let $F_{\mathrm{o}}\left(G_{1}\right)=w_{1}, w_{2}, \cdots, w_{t}$, $w_{1}=v_{1}$, and $w_{t}=v_{2}$. We draw $G_{1}$ as illustrated in Fig. 6, depending on whether $\left(v_{1}, v_{2}\right)$ is a real edge or not, and $w_{2} \in$ $V_{l}$ or not.

\section{Initialize:}

Case 1: $v_{1}$ and $v_{2}$ are adjacent in the original graph $G$, that is, $\left(v_{1}, v_{2}\right)$ is a real edge (see Figs. 6 (a) and (b)).

Set $P\left(w_{1}\right)=(0,0)$;

Case 1(a): $w_{2} \in V_{l}$ (see Fig. 6 (a)).

Set $P\left(w_{i}\right)=(i-3,1)$ for each $i, 2 \leq i \leq t-1$;

Set $P\left(w_{t}\right)=(t-2,0)$;

Case 1(b): $w_{2} \notin V_{l}$ (see Fig. 6(b)).

Set $P\left(w_{i}\right)=(i-1,1)$ for each $i, 2 \leq i \leq t-1$;

Set $P\left(w_{t}\right)=(t, 0)$;

Case 2: Otherwise, that is, $\left(v_{1}, v_{2}\right)$ is a virtual edge (see Fig. $6(\mathrm{c}))$.

Set $P\left(w_{i}\right)=(i-1,0)$ for each $i, 1 \leq i \leq t$.

We then extend a drawing $D_{k-1}$ of $G_{k-1}$ to a drawing $D_{k}$ of $G_{k}$ for each index $k, 2 \leq k \leq m$. For each $k, 2 \leq k \leq m$, let $F_{\mathrm{o}}\left(G_{k-1}\right)=w_{1}, w_{2}, \cdots, w_{t}$, where $w_{1}=v_{1}, w_{t}=v_{2}$, and $w_{1}, w_{2}, \cdots, w_{t}$ appear clockwise on $F_{\mathrm{o}}\left(G_{k-1}\right)$ in this order, as illustrated in Fig. 7. Let $U_{k}=\left\{u_{1}, u_{2}, \cdots, u_{l}\right\}$. Let $w_{p}$ be the leftmost neighbor of $u_{1}$, and let $w_{q}$ be the rightmost neighbor of $u_{l}$. Let $w_{f}$ be the vertex with the maximum index $f$ among all the vertices $w_{i}, 1 \leq i \leq t$, on $F_{\mathrm{o}}\left(G_{k-1}\right)$ that are contained 

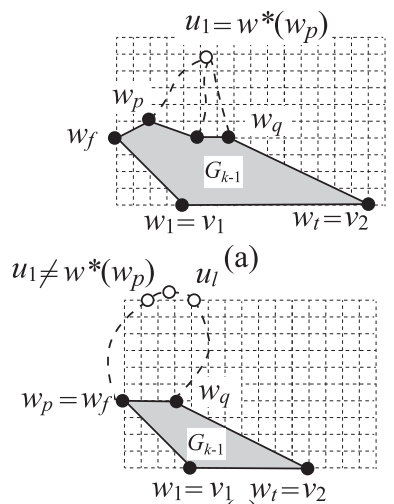

(c)

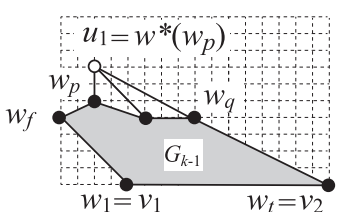

(b)

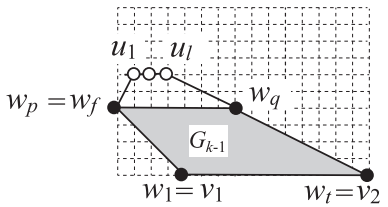

(d)
Fig. 7 Graphs (a) $G_{k-1}$ and (b) $G_{k}$ when Case $x\left(w_{q}\right)-x\left(w_{p}\right)+\epsilon$ is an odd number, graphs (c) $G_{k-1}$ and (d) $G_{k}$ when Case $x\left(w_{q}\right)-x\left(w_{p}\right)+\epsilon$ is an even number.

in $V_{l}$.

Assume that a drawing $D_{k-1}$ of $G_{k-1}$ satisfies the following five conditions (sh'1)-(sh'5).

(sh'1) $P\left(w_{1}\right)=(0,0), x\left(w_{t}\right) \leq 2\left|V\left(G_{k-1}\right)\right|$ and $y\left(w_{t}\right)=0$.

(sh'2) $x\left(w_{1}\right)>x\left(w_{2}\right)>\cdots>x\left(w_{f}\right), x\left(w_{f}\right) \leq x\left(w_{f+1}\right) \leq$ $\cdots \leq x\left(w_{t}\right)$, where $x\left(w_{i}\right)$ is the $x$-coordinate of $w_{i}$.

(sh'3) Every edge $\left(w_{i}, w_{i+1}\right), 1 \leq i \leq t-1$, has slope -1 , $-1 / 2,0$ or $[1,+\infty]$.

(sh'4) Every inner face of $G_{k-1}$ is drawn as a convex polygon.

(sh'5) Vertex $w_{i}, 2 \leq i \leq t-1$, has one or more neighbors in $\overline{G_{k-1}}$ if $w_{i}$ is a convex apex.

Indeed $D_{1}$ satisfies the five conditions above. We extend $D_{k-1}$ to $D_{k}$ so that $D_{k}$ satisfies them, as follows.

In our algorithm, we wish to put the vertex $u_{1}$ of $U_{k}$ on a grid point so that the edge $\left(w_{p}, u_{1}\right)$ has slope either -1 (if $u_{1} \in V_{l}$ ) or $[1,+\infty]$ (otherwise) and put the vertex $u_{l}$ on a grid point so that the edge $\left(u_{l}, w_{q}\right)$ has slope $-1 / 2$. Furthermore, if $\left|U_{k}\right| \geq 2$, then we wish to put the vertices $u_{2}, u_{3}, \cdots, u_{l-1}$ so that, for each $i, 1 \leq i \leq l-1$, the edge $\left(u_{i}, u_{i+1}\right)$ has slope 0 and the distance between two vertices $u_{i}$ and $u_{i+1}$ is equal to 1 . For this purpose, before installing $U_{k}=\left\{u_{1}, u_{2}, \cdots, u_{l}\right\}$ to $D_{k-1}$, we shift some vertices of $G_{k-1}$ to the right as illustrated in Figs. 7 (a)-(d).

Let $\epsilon$ be 0 if $u_{1}=w^{*}\left(w_{p}\right)$, and 1 otherwise. If $x\left(w_{q}\right)-x\left(w_{p}\right)+\epsilon$ is an odd number, as illustrated in Fig. 7 (a), then we shift $w_{q}, w_{q+1}, \cdots, w_{t}$ of $G_{k-1}$ and some inner vertices of $G_{k}$ to the right by distance $\left|U_{k}\right|$, as illustrated in Fig. 7 (b). Otherwise $\left(x\left(w_{q}\right)-x\left(w_{p}\right)+\epsilon\right.$ is an even number, as illustrated in Fig. 7 (c)), we shift $w_{q}, w_{q+1}, \cdots, w_{t}$ of $G_{k-1}$ and some inner vertices of $G_{k}$ to the right by distance $\left|U_{k}\right|+1$, as illustrated in Fig. 7 (d).

Then, we install $U_{k}$ to $D_{k-1}$ as follows.

\section{Install $U_{k}$ :}

Case 1: $u_{1} \in V_{l}$.

For each $i, 1 \leq i \leq l$, we set

$$
x\left(u_{i}\right)=2 x\left(w_{p}\right)-x\left(w_{q}\right)+2 y\left(w_{p}\right)-2 y\left(w_{q}\right)
$$

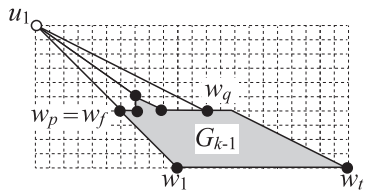

(a)

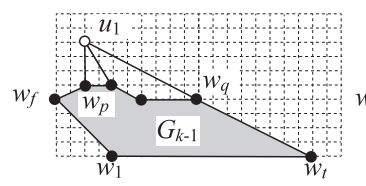

(c)

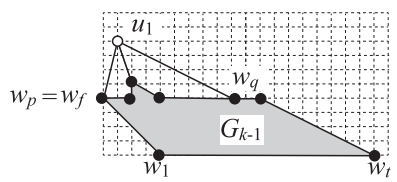

(e)

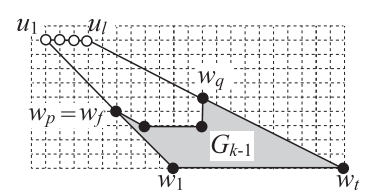

(b)

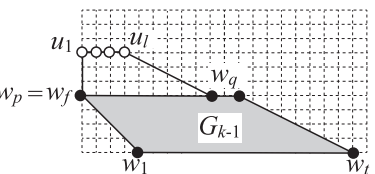

(d)

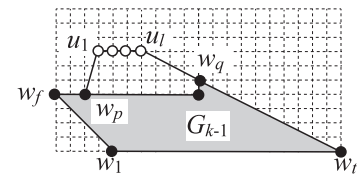

(f)
Fig. 8 Installing $U_{k}$ to $D_{k-1}$.

$$
+l-2+i
$$

and set

$$
y\left(u_{i}\right)=-x\left(w_{p}\right)+x\left(w_{q}\right)-y\left(w_{p}\right)+2 y\left(w_{q}\right)-l+1,
$$

as illustrated in Figs. 8 (a), (b);

Case 2: Otherwise.

For each $i, 1 \leq i \leq l$, we set

$$
x\left(u_{i}\right)=x\left(w_{p}\right)+i-1+\epsilon,
$$

and set

$$
y\left(u_{i}\right)=y\left(w_{q}\right)-\left(x\left(w_{p}\right)-x\left(w_{q}\right)+l-1+\epsilon\right) \times 1 / 2,
$$

as illustrated in Figs. 8 (c), (d) for the case $\epsilon=0$ and in Figs. 8 (e), (f) for the case $\epsilon=1$.

Clearly, the drawing $D_{k}$ of $G_{k}$ extended from $D_{k-1}$ satisfies conditions (sh'1), (sh'2) and (sh'3). One can prove similarly as in [2] that $D_{k}$ satisfies conditions (sh'4) and (sh'5). By the condition (sh'1), we have $x\left(v_{2}\right) \leq 2 n$ in $D_{m}$ of $G_{m}=G$ and hence one can easily show that the triangular drawing algorithm yields a convex grid drawing of $G$ on a $W \times H$ grid with $W \leq 4 n$ and $H \leq 2 n$. Furthermore, one can easily show that the triangular drawing algorithm takes linear time.

We thus have the following lemma.

Lemma 6: For a plane graph $G$ having a canonical decomposition $\Pi=\left(U_{1}, U_{2}, \cdots, U_{m}\right)$ with respect to $v_{1}, v_{2}$ and $v_{n}$, the triangular drawing algorithm yields a convex grid drawing of $G$ on a $W \times H$ grid with $W \leq 4 n$ and $H \leq 2 n$ in linear time.

\section{Convex Grid Drawing Algorithm}

In this section, we present a linear-time algorithm to find a convex grid drawing $D$ of an internally triconnected plane 


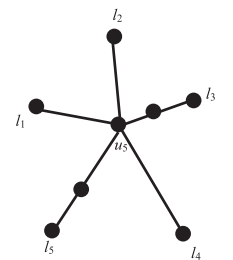

(a) (b)

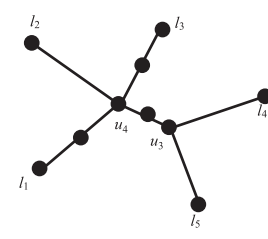

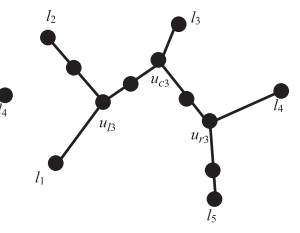

(c)
Fig. 9 Decomposition trees $T(G)$ (a) having a node of degree five, (b) having a node of degree four and a node of degree three, and (c) having three nodes of degree three.

graph $G$ whose decomposition tree $T(G)$ has exactly five leaves. Such a graph $G$ does not have a canonical decomposition, and hence none of the algorithms in [1], [2], [6] and [9] and the pentagonal drawing algorithm in Sect. 3 can find a convex grid drawing of $G$. Our algorithm draws the outer facial cycle $F_{\mathrm{o}}(G)$ as a pentagon as illustrated in Fig. $1(\mathrm{~d})$. The algorithm first divides $G$ into a left subgraph $G_{l}$, a right subgraph $G_{r}$ and a center subgraph $G_{c}$ as illustrated in Fig. 1 (b), then draw $G_{l}$ and $G_{r}$ by using the triangular drawing algorithm in Sect. 4 and draw $G_{c}$ by using a modified pentagonal drawing algorithm in Sect. 5.4 as illustrated in Figs. 1 (c), and finally combine these three drawings to a convex grid drawing of $G$ as illustrated in Fig. 1 (d).

\subsection{Division}

We first explain how to divide $G$ into $G_{l}, G_{r}$ and $G_{c}$. (See Figs. 1 (a) and (b).)

One may assume that the five leaves $l_{1}, l_{2}, l_{3}, l_{4}$ and $l_{5}$ of $T(G)$ appear clockwise in $T(G)$ in this order, as illustrated in Fig. 9. Clearly, there are three cases to consider.

Case a: exactly one node $u_{5}$ of $T(G)$ has degree five and each of the other non-leaf nodes has degree two as illustrated in Fig. 9 (a).

Case b: exactly one node $u_{4}$ has degree four, exactly one node $u_{3}$ has degree three and each of the other non-leaf nodes has degree two as illustrated in Fig. 9 (b).

Case c: exactly three nodes $u_{l 3}, u_{c 3}$ and $u_{r 3}$ have degree three and each of the other non-leaf nodes has degree two as illustrated in Fig. 9 (c). similar.

We only consider Case a, because the other cases are

As the five apices of the pentagonal contour of $G$, we choose five outer vertices $a_{i}, 1 \leq i \leq 5$ of $G$; let $a_{i}$ be an arbitrary outer vertex in the component corresponding to leaf $l_{i}$ that is not a vertex of the separation pair of the component. The five vertices $a_{1}, a_{2}, a_{3}, a_{4}$ and $a_{5}$ appear clockwise on $F_{\mathrm{o}}(G)$ in this order as illustrated in Fig. 1 (a).

Let $P\left(l_{i}\right), 1 \leq i \leq 5$, be a path from $l_{i}$ to $u_{5}$ in $T(G)$. We choose arbitrary two nonconsecutive leaves $l_{i}$ and $l_{i+2}$, where indices are computed as modulo 5. We then split the graphs corresponding to $P\left(l_{i}\right)-u_{5}$ and corresponding to $P\left(l_{i+2}\right)-u_{5}$ from $G$. Let $G_{l}$ be the graph corresponding to $P\left(l_{i}\right)-u_{5}$, let $G_{r}$ be the graph corresponding to $P\left(l_{i+2}\right)-u_{5}$

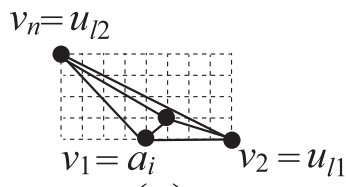

(a)

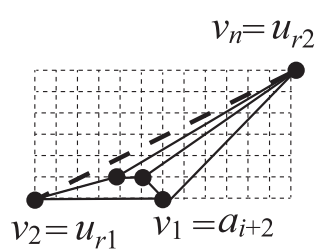

(c)

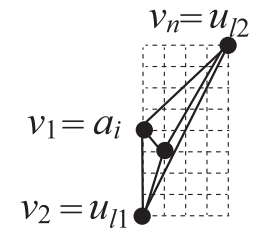

(b)

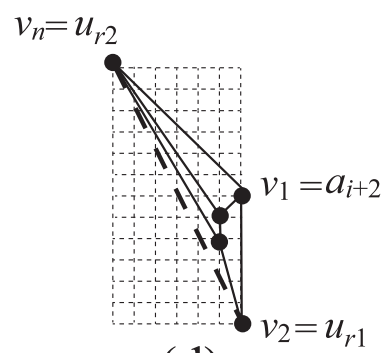

(d)
Fig. 10 (a) A triangular drawing of $G_{l}$, (b) a drawing $D_{l}$ of $G_{l}$, (c) a triangular drawing of $G_{r}$, and (d) a drawing $D_{r}$ of $G_{r}$.

and let $G_{c}$ be the graph corresponding to $G-G_{l}-G_{r}$. In Fig. 1 (b), $G_{l}$ is the graph corresponding to $P\left(l_{1}\right)-u_{5}$, and $G_{r}$ is the graph corresponding to $P\left(l_{3}\right)-u_{5}$. Let $\left\{v_{l_{1}}, v_{l_{2}}\right\}$ be the separation pair for $G_{l}$ and $G_{c}$, let $\left\{v_{r_{1}}, v_{r_{2}}\right\}$ be the separation pair for $G_{r}$ and $G_{c}$ and let $v_{l_{1}}, v_{l_{2}}, v_{r_{2}}$ and $v_{r_{1}}$ appear around $F_{\mathrm{o}}(G)$ in this order, as illustrated in Fig. 1 (a). If $\left(v_{l_{1}}, v_{l_{2}}\right)$ (resp. $\left.\left(v_{r_{1}}, v_{r_{2}}\right)\right)$ is a real edge of $G$, then let $G_{l}$ (resp. $\left.G_{r}\right)$ and $G_{c}$ be the graphs obtained by deleting a virtual edge $\left(v_{l_{1}}, v_{l_{2}}\right)$ (resp. $\left.\left(v_{r_{1}}, v_{r_{2}}\right)\right)$ from each graph defined above.

\subsection{Drawing of $G_{l}$}

We obtain a triangular drawing of $G_{l}$ by using the triangular drawing algorithm in Sect. 4 as $v_{1}=a_{i}, v_{2}=v_{l_{1}}$ and $v_{n}=v_{l_{2}}$, respectively, as illustrated in Fig. 10 (a). We finally rotate the drawing by $90^{\circ}$ clockwise and obtain a drawing $D_{l}$ of $G_{l}$, as illustrated in Fig. 10 (b).

\subsection{Drawing of $G_{r}$}

Let $G_{r}^{\prime}$ be a "mirror" copy of $G_{r}$. We obtain a triangular drawing of $G_{r}^{\prime}$ by using the triangular drawing algorithm in Sect. 4 as $v_{1}=a_{i+2}, v_{2}=v_{r_{1}}$ and $v_{n}=v_{r_{2}}$, respectively. We then modify the drawing of $G_{r}^{\prime}$ using the left-right reflection and we obtain a triangular drawing of $G_{r}$, as illustrated in Fig. 10 (c). We finally rotate the drawing by $90^{\circ}$ counterclockwise and obtain a drawing $D_{r}$ of $G_{r}$, as illustrated in Fig. 10 (d).

\subsection{Drawing of $G_{c}$}

In this section, we present a linear-time algorithm, called a heptagonal drawing algorithm to find a convex grid drawing of $G_{c}$ with a heptagonal outer polygon. This algorithm finds a convex grid drawing of $G_{c}$ whose outer polygon is a heptagon with apices $v_{1}, v_{2}, v_{r_{1}}, v_{r_{2}}, v_{n}, v_{l_{2}}$ and $v_{l_{1}}$. We modify 
the pentagonal drawing algorithm in Sect. 3, as follows.

We use the pentagonal drawing algorithm as $v_{1}=a_{i+4}$, $v_{2}=a_{i+3}, v_{n}=a_{i+1}, v_{l}=v_{l_{2}}$ and $v_{r}=v_{r_{2}}$. Actually, the method deciding the coordinates of each vertex other than $v_{l_{2}}$ and $v_{r_{2}}$ is quite similar to the pentagonal drawing algorithm. Thus we will explain how to decide the coordinates of $v_{l_{2}}$ and $v_{r_{2}}$. We only consider the case for $v_{l_{2}}$. The case for $v_{r_{2}}$ is similar.

If $v_{l_{2}} \in U_{k}$, then $v_{l_{2}}$ should be the first vertex of $U_{k}$. Let $U_{k}=\left\{v_{l_{2}}=u_{1}, u_{2}, \cdots, u_{l}\right\}$, let $w_{p}$ be the leftmost neighbor of $v_{l_{2}}=u_{1}$, and let $w_{q}$ be the rightmost neighbor of $u_{l}$. Then $w_{p}=v_{l_{1}}$, of course.

We first shift $w_{1}, w_{2}, \cdots, w_{p}$ of $G_{k-1}$ and some inner vertices of $G_{k}$ to the left by distance $\left|U_{k}\right|$, and then shift $w_{q}, w_{q+1}, \cdots, w_{t}$ of $G_{k-1}$ and some inner vertices of $G_{k}$ to the right by distance $\left|U_{k}\right|$. After the operation, we shift all vertices of $G_{k-1}$ to the right by distance $\left|U_{k}\right|$ so that $P\left(w_{1}\right)=(0,0)$.

Let $D_{m}$ be the Manhattan distance between $w_{p}$ and $w_{q}$. We wish to put vertices $U_{k}=\left\{v_{l_{2}}=u_{1}, u_{2}, \cdots, u_{l}\right\}$ so that the edge $\left(w_{p}, u_{1}\right)=\left(v_{l_{1}}, v_{l_{2}}\right)$ has slope +2 and the edge $\left(u_{l}, w_{q}\right)$ has slope -1 . Furthermore, if $\left|U_{k}\right| \geq 2$, then we wish to put vertices $u_{2}, u_{3}, \cdots, u_{l-1}$ so that, for each $i, 1 \leq i \leq l-1$, the edge $\left(u_{i}, u_{i+1}\right)$ has slope 0 and the distance between two vertices $u_{i}$ and $u_{i+1}$ is equal to 2 . Then $D_{m}-2\left(\left|U_{k}\right|-1\right)$ should be a multiple of 3 , that is, $\left(D_{m}-2\left(\left|U_{k}\right|-1\right)\right) \bmod 3=$ 0 and hence we will do some additional shift operations if $D_{m} \bmod 3 \neq 0$. We have the following two cases to consider.

Case (I): $\left(D_{m}-2\left(\left|U_{k}\right|-1\right)\right) \bmod 3=1$.

In this case, we shift $w_{q}, w_{q+1}, \cdots, w_{t}$ of $G_{k-1}$ and some inner vertices of $G_{k}$ to the right by distance 2 .

Case (II): $\left(D_{m}-2\left(\left|U_{k}\right|-1\right)\right) \bmod 3=2$.

In this case, we shift $w_{q}, w_{q+1}, \cdots, w_{t}$ of $G_{k-1}$ and some inner vertices of $G_{k}$ to the right by distance 1 .

Then, $D_{m}-2\left(\left|U_{k}\right|-1\right)$ becomes a multiple of 3 and hence the straight line with slope +2 through $\left(x\left(w_{p}\right), y\left(w_{p}\right)\right)$ and the straight line with slope -1 through $\left(x\left(w_{q}\right)-2\left(\left|U_{k}\right|-\right.\right.$ 1), $\left.y\left(w_{q}\right)\right)$ intersect at a grid point, which is denoted by $P$.

In Sect. 5.5, we will combine the drawing $D_{l}$ of the left subgraph $G_{l}$ and the drawing $D_{c}$ of the center subgraph $G_{c}$ so that the edge $\left(v_{l_{1}}, v_{l_{2}}\right)$ of $G_{l}$ overlaps the same one of $G_{c}$. If the length of the edge $\left(v_{l_{1}}, v_{l_{2}}\right)$ of $G_{l}$ is not equal to the length of the edge $\left(v_{l_{1}}, v_{l_{2}}\right)$ of $G_{c}$, then we widen the narrower one by the shift operation so that both have the same length. We have the two cases to consider.

Case (i): $H\left(D_{l}\right)>y(P)-y\left(w_{p}\right)$. (See Fig. 11 (a).)

In this case, we shift $w_{q}, w_{q+1}, \cdots, w_{t}$ of $G_{k-1}$ and some inner vertices of $G_{k}$ to the right by distance $\left(H\left(D_{l}\right)-\right.$ $\left.\left(y(P)-y\left(w_{p}\right)\right)\right) \times 3 / 2$. In Fig. $11(\mathrm{a}), H\left(D_{l}\right)-(y(P)-$ $\left.y\left(w_{p}\right)\right)$ is equal to 2 and hence we shift by distance 3 , as illustrated in Fig. 11 (b). Since $H\left(D_{l}\right)-\left(y(P)-y\left(w_{p}\right)\right)$ is an even number, $\left(H\left(D_{l}\right)-\left(y(P)-y\left(w_{p}\right)\right)\right) \times 3 / 2$ is a multiple of 3 and hence $D_{m}-2\left(\left|U_{k}\right|-1\right)$ is still a multiple of 3 .

Case (ii): $H\left(D_{l}\right) \leq y(P)-y\left(w_{p}\right)$. (See Fig. $11(\mathrm{c})$.)

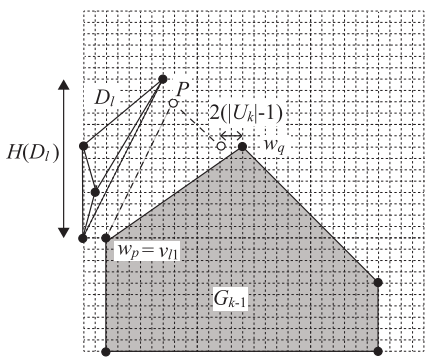

(a)

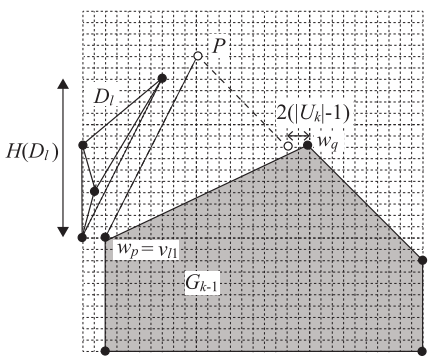

(c)

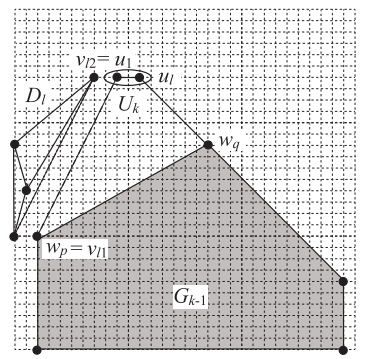

(b)

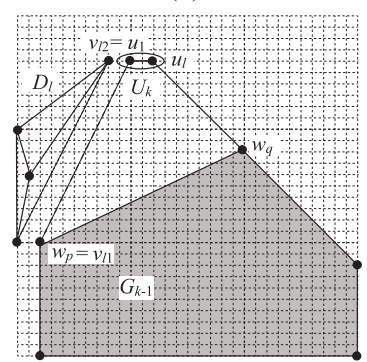

(d)
Fig. 11 Illustrations for (a),(b) Case (i), and (c),(d) Case (ii).

In this case, we extend the drawing $D_{l}$ so that the length of the edge $\left(v_{l_{1}}, v_{l_{2}}\right)$ of $G_{l}$ is equal to the length between $P\left(w_{p}\right)$ and $P$, as illustrated in Fig. $11(\mathrm{~d})$.

We then put vertices in $U_{k}$ so that the edge $\left(v_{l_{1}}, v_{l_{2}}\right)$ has slope +2 and the edge $\left(u_{l}, w_{q}\right)$ has slope -1 . Furthermore, if $\left|U_{k}\right| \geq 2$, then we put vertices $u_{2}, u_{3}, \cdots, u_{l-1}$ so that the edges $\left(u_{i}, u_{i+1}\right)$ has slope 0 and the distance between two vertices $u_{i}$ and $u_{i+1}$ is equal to 2 , for each $i, 1 \leq i \leq l-1$, as illustrated in Figs. 11 (b) and (d).

One can prove similarly as in [11] that the heptagonal drawing algorithm yields a convex grid drawing of $G_{c}$. We finally consider the width $W\left(D_{c}\right)$ and the height $H\left(D_{c}\right)$ of the drawing $D_{c}$ of $G_{c}$.

Let $n_{l}$ be the number of vertices of $G_{l}$, let $n_{r}$ be the number of vertices of $G_{r}$ and let $n_{c}$ be the number of vertices of $G_{c}$. Then $n_{l}+n_{r}+n_{c}=n+4$, of course. Since every vertex of a plane graph $G$ has degree three or more, each component corresponding to $l_{i}$ of $T(G)$, for each $i, 1 \leq i \leq 5$ has four or more vertices and hence we have $n_{l}, n_{r} \geq 4$ and $n_{c} \geq 10$. Since we may shift by distance $2+H\left(D_{l}\right) \times 3 / 2$ for $v_{l_{2}}$ and by distance $2+H\left(D_{r}\right) \times 3 / 2$ for $v_{r_{2}}$, we have

$$
W\left(D_{c}\right) \leq 2 n_{c}+4+H\left(D_{l}\right) \times 3 / 2+H\left(D_{r}\right) \times 3 / 2 \text {. }
$$

By Lemma 6 and the algorithms in Sects. 5.2 and 5.3, we have $H\left(D_{l}\right) \leq 4 n_{l}$ and $H\left(D_{r}\right) \leq 4 n_{l}$ and hence

$$
W\left(D_{c}\right) \leq 2 n_{c}+4+6 n_{l}+6 n_{r} .
$$

Since $n_{l}+n_{r}+n_{c}=n+4$ and $n_{c} \geq 10$, we have

$$
W\left(D_{c}\right) \leq 6\left(n_{l}+n_{r}+n_{c}\right)+4-4 n_{c} \leq 6 n .
$$

Furthermore, one can prove similarly as in [11] that $H\left(D_{c}\right) \leq n^{2}$.

We thus have the following lemma. 
Lemma 7: For a plane graph $G_{c}$ having a canonical decomposition $\Pi=\left(U_{1}, U_{2}, \cdots, U_{m}\right)$ with respect to $v_{1}, v_{2}$ and $v_{n}$, the heptagonal drawing algorithm yields a convex grid drawing of $G_{c}$ on a $W \times H$ grid with $W \leq 6 n$ and $H \leq n^{2}$ in linear time.

\subsection{Drawing of $G$}

We first arrange $D_{c}$ so that $x\left(a_{i+4}\right)=0$ and $y\left(a_{i+4}\right)=0$. We then arrange $D_{l}$ so that the edge $\left(v_{l_{1}}, v_{l_{2}}\right)$ of $G_{l}$ overlaps the same one of $G_{c}$. We also arrange $D_{r}$ so that the edge $\left(v_{r_{1}}, v_{r_{2}}\right)$ of $G_{r}$ overlaps the same one of $G_{c}$. We finally remove the edges $\left(v_{l_{1}}, v_{l_{2}}\right)$ and $\left(v_{r_{1}}, v_{r_{2}}\right)$ if they are not original edges of $G$, as illustrated in Fig. 1 (d).

\subsection{Validity of Drawing Algorithm}

In this section, we show that the drawing $D$ obtained above is a convex grid drawing of $G$. By Lemma 6 , both $D_{l}$ and $D_{r}$ are convex grid drawings. Furthermore, by Lemma 7, $D_{c}$ is also a convex grid drawing. Therefore, one can easily show that $D$ is a convex grid drawing of $G$ with pentagonal contour. Clearly, the size of the grid of the drawing $D$ of $G$ is equal to the size of $D_{c}$ of $G_{c}$ and hence, by Lemma 7, we have $W(D) \leq 6 n$ and $H(D) \leq n^{2}$.

We thus have the following theorem.

Theorem 1: Assume that $G$ is an internally triconnected plane graph, every vertex of $G$ has degree three or more, and the triconnected component decomposition tree $T(G)$ has exactly five leaves. Then our algorithm finds a convex grid drawing of $G$ on a $6 n \times n^{2}$ grid in linear time.

\section{Conclusions}

In this paper, we showed that every internally triconnected plane graph $G$ whose decomposition tree $T(G)$ has exactly five leaves has a convex grid drawing on a $6 n \times n^{2}$ grid, and we present a linear-time algorithm to find such a drawing. This is the first algorithm that finds a convex grid drawing of such a graph $G$ on a grid of polynomial size. The remaining problem is to obtain an algorithm for an internally triconnected plane graph whose decomposition tree has six or more leaves.

\section{Acknowledgements}

This work was supported by JSPS KAKENHI Grant Number 23700008. We thank Satoko Chiba for fruitful discussions.

\section{References}

[1] N. Bonichon, S. Felsner, and M. Mosbah, "Convex drawings of 3connected plane graphs," Algorithmica, vol.47, no.4, pp.399-420, 2007.

[2] M. Chrobak and G. Kant, "Convex grid drawings of 3-connected planar graphs," International Journal of Computational Geometry and Applications, vol.7, pp.211-223, 1997.

[3] N. Chiba, K. Onoguchi, and T. Nishizeki, "Drawing planar graphs nicely," Acta Inform., vol.22, pp.187-201, 1985.

[4] N. Chiba, T. Yamanouchi, and T. Nishizeki, "Linear algorithms for convex drawings of planar graphs," in Progress in Graph Theory, ed. J.A. Bondy and U.S.R. Murty, pp.153-173, Academic Press, 1984.

[5] H. de Fraysseix, J. Pach, and R. Pollack, "How to draw a planar graph on a grid," Combinatorica, vol.10, pp.41-51, 1990.

[6] S. Felsner, "Convex drawings of plane graphs and the order dimension of 3-polytopes," Order, vol.18, pp.19-37, 2001.

[7] J.E. Hopcroft and R.E. Tarjan, "Dividing a graph into triconnected components," SIAM J. Compt., vol.2, no.3, pp.135-158, 1973.

[8] T. Hashimoto, K. Miura, and T. Nishizeki, "Convex grid drawings of internally triconnected plane graphs," IEICE Trans. Inf. \& Syst. (Japanese Edition), vol.J95-D, no.3, pp.356-365, March 2012.

[9] K. Miura, M. Azuma, and T. Nishizeki, "Canonical decomposition, realizer, Schnyder labeling and orderly spanning trees of plane graphs," International Journal of Foundations of Computer Science, vol.16, no.1, pp.117-141, 2005.

[10] K. Miura, M. Azuma, and T. Nishizeki, "Convex drawings of plane graphs of minimum outer apices," International Journal of Foundations of Computer Science, vol.17, no.5, pp.1115-1127, 2006.

[11] K. Miura, A. Kamada, and T. Nishizeki, "Convex grid drawings of plane graphs with rectangular contours," Journal of Graph Algorithms and Applications, vol.12, no.2, pp.197-224, 2008.

[12] T. Nishizeki and M.S. Rahman, Planar Graph Drawing, World Scientific, Singapore, 2004.

[13] C. Thomassen, "Planarity and duality of finite and infinite graphs," J. Combinatorial Theory, Series B, vol.29, pp.244-271, 1980.

[14] W.T. Tutte, Connectivity in Graphs, University of Toronto Press, Toronto, 1966.

[15] X. Zhou and T. Nishizeki, "Convex drawings of internally triconnected plane graphs on $O\left(n^{2}\right)$ grids," Discrete Mathematics, Algorithms and Applications, vol.2, pp.347-362, 2009.

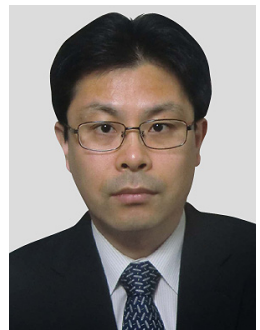

Kazuyuki Miura received the B.E., M.E., and Ph.D. degrees from Tohoku University, Sendai, Japan, in 1996, 1998 and 2001, respectively. He was a research associate at Tohoku University during 2001-2004. He joined the Faculty of Symbiotic Systems Science, Fukushima University, as an Associate Professor in 2005. His research interests are in the field of algorithms for planar graphs and graph drawing. He is a member of IEICE and IPSJ. 